\title{
Da Génese do Termo Química Verde às Colorações Discrepantes da Química e da BioteCnOLOGIA
}

\author{
AdéLIo A. S. C. Machado*
}

Discute-se a origem do termo Química Verde e apresentam-se, comparativamente, as definições de Química Verde, Vermelha e Preta, e de Biotecnologia Verde, Vermelha, Preta, Azul e Branca, para chamar a atenção para o facto de os "códigos de coloração" daqueles dois ramos da ciência e tecnologia serem diferentes - e tentar prevenir confusões. Discute-se a razão que poderá ter conduzido à discrepância, concluindo-se hipoteticamente que o uso de cores diversas se fundamenta numa base cultural diferente, o que dificultará quaisquer esforços de unificação.

Esta ligeira (e colorida) nota descreve a origem do termo Química Verde $(\mathrm{QV})^{1}$ e analisa a discrepância do uso das cores entre a Química e a Biotecnologia para designar actividades nos respectivos âmbitos. Tem um primeiro objectivo, pragmático, de alertar os leitores para as diferentes colorações das duas ciências e prevenir eventuais confusões; e um segundo, lateral, de tentar chegar a uma explicação para a diferença.

Química Verde. Aparentemente, o termo QV começou a ser usado nos princípios dos anos noventa, nos EUA, na sequência da promulgação da primeira peça de legislação ambiental de prevenção proactiva da poluição (o "Pollution Prevention Act", 1990), que prescrevia uma nova atitude de prevenção na fonte - não produzir substâncias tóxicas para não ter problemas de poluição a remediar. O termo QV terá sido introduzido por químicos do "Office of Pollution Prevention and Toxics" (OPPT) da "Environmental Protection Agengy" (US EPA), no âmbito de um programa de pesquisa destinado a potenciar a referida legislação. Este programa visava incentivar a investigação do design de vias de síntese inovadoras quanto à diminuição intencional da quantidade de resíduos produzidos ("Alternative Synthetic Design for Pollution Prevention") - e introduziu o conceito de síntese benigna por design ("benign by design") e a sigla $\mathrm{P}_{2}$ ("Pollution Prevention"). É interessante notar que nas actas de um primeiro simpó-

\footnotetext{
LAQUIPAI, Departamento de Química da Faculdade de Ciências da Universidade do Porto

R. Campo Alegre, 687 - 4169-007 Porto

amachado@fc.up.pt
}

sio científico realizado no âmbito do programa, em 1993, publicadas pela "American Chemical Society" (ACS) [1], o termo QV não é usado, excepto num In Memoriam em homenagem a K. G. Hancock, acrescentado a posteriori, em que se refere a importância da actividade deste investigador para o lançamento da QV - no título e no texto o termo usado é sempre síntese benigna por design. Em contraste, nas publicações subsequentes com a mesma génese, o termo QV já é usado nos títulos, o que sugere a sua aceitação, plena (e rápida), pelos proponentes norte-americanos da nova postura na prática da química [2-7]. Por outro lado, em 1993, o referido programa de pesquisa passou a ser designado pela US EPA por "US Green Chemistry Program" [8].

Fora deste círculo, o nome QV começou a ser usado de forma coloquial (jornais, encontros, etc.) [9], mas encontrou inicialmente uma certa resistência à penetração na literatura científica e pedagógica, possivelmente devido à conotação política que a palavra verde tinha então em muitos países [10] - estava ligada à primeira geração dos modernos ambientalistas, "os verdes" puros e duros, cujo objectivo era a conservação do ambiente a todo o custo, independentemente das consequências económicas e sociais. ${ }^{2}$

Em revistas científicas, o termo parece ter surgido pela primeira vez em 1993 no título de um comentário publicado na revista Science [11]; em revistas com objectivos pedagógicos, o primeiro artigo que inclui o termo no título foi publicado em 1995, no
Journal of Chemical Education (JCEd) [12]. No entanto, nos anos que se seguiram, a utilização do termo em revistas dos dois tipos foi escasso a situação só se alterou quando em 1999, a "Royal Society of Chemistry" (RSC) iniciou a publicação da revista Green Chemistry [13]. Na realidade, buscas realizadas nas páginas de publicações científicas da ACS [14] e do JCEd [15] mostram que o termo QV só ganhou aceitação científica e pedagógica a partir de 2000 (ver a Fig.1). Uma busca semelhante na página das revistas da RSC [16] revelou que o termo foi usado pela primeira vez em 1998 (1 artigo), vulgarizando-se depois em consequência da publicação da referida revista; ou seja, os resultados foram análogos aos da Fig. 1 , não sendo incluídas nela porque o modo como as referências são apresentadas nos resultados da pesquisa nem sempre permite identificar o tipo das peças bibliográficas encontradas (num total de 136 entre 1998 e 2008 - em pré-publicação). Outra busca do mesmo tipo no ScienceDirect (revistas da Elsevier) [17] revelou também uma situação semelhante - em 300 peças bibliográficas encontradas, só 9 foram publicadas entre 1997 e 2000 , exclusive, mas o ritmo de publicações subiu depois rapidamente. ${ }^{3}$

Em resumo, a paternidade do termo QV é elusiva [9], e as pesquisas bibliográficas efectuadas mostram que o termo só se tornou vulgar no terceiro milénio, quando já tinha ganho aceitação a ideia de que os problemas do ambiente tinham de ser resolvidos num âmbito alargado que englobava também os problemas societários e económicos - o do Desenvolvimento 
Sustentável. Este facto sugere que o tom do verde da QV não é o mesmo do primitivo ambientalismo (ver atrás). A designação $Q V$ é vigorosa, não espantando que tenha ganhado aceitação crescente, quer nos EUA quer na Europa, embora o conceito que Ihe está subjacente, muito complexo, tenha ainda de ser clarificado.

mo químico. A cor vermelha é usada aqui, como nas luzes de tráfico, em associação com proibição de actividade, neste caso actividade química para praticar o mal; e em contraste com o verde de $\mathrm{QV}$, interpretado como significando que esta pratica proactivamente o bem, desequilibrando a natureza dual ${ }^{4}$ da química no sentido deste - e suportando a sua aceitação.

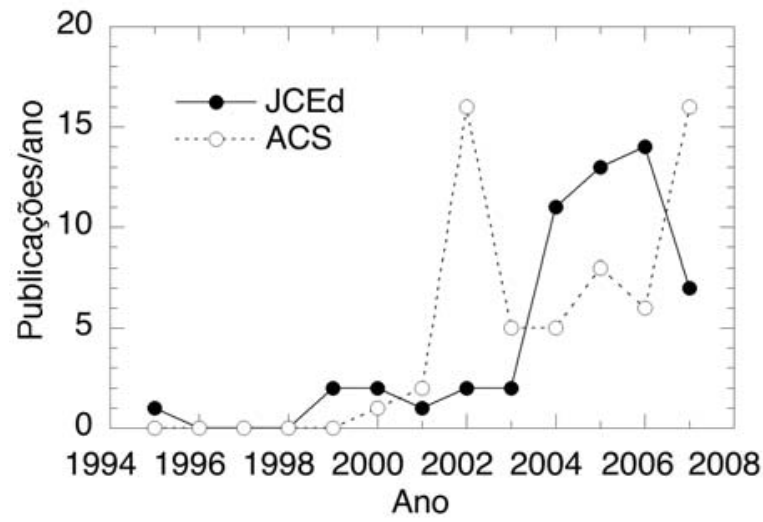

Figura 1 Artigos com menção de QV no título e resumo publicados anualmente nos EUA (Foi pesquisado o termo "green chemistry" nos títulos e resumos de artigos (ACS) ou nos títulos e palavras-chave (JCEd), mas excluídas recensões de livros e notícias. O número elevado de artigos científicos (ACS) em 2002 resultou da publicação de números especiais sobre OV pelas revistas Accounts of Chemical Research e Industrial and Engineering Chemistry Research.)

Química Vermelha e Química Preta. Estes dois termos foram usados numa conferência sobre catálise, em 1999, por G. Ourisson, Presidente da Academia de Ciências Francesa [10], para designar duas características da Química Industrial que a QV visa eliminar. A Química Preta dizia respeito à produção de resíduos e poluição - o adjectivo preto foi sugerido pelo intenso fumo negro das chaminés das fábricas da Revolução Industrial. Por outro lado, a Química Vermelha evocava os efeitos nocivos da química para a segurança e saúde humana, bem evidenciados por desastres brutais ao longo da história da Indústria Química - por exemplo, os particularmente nocivos de Oppau (Alemanha, 1921), Texas City (EUA, 1947) e Bhopal (Índia, 1984). Segundo o referido conferencista, o objectivo da prática da QV pela Química Industrial seria eliminar estes aspectos deletérios da sua actividade [10].

Nenhum dos dois termos se vulgarizou mas, recentemente (2005) [18], o nome Química Vermelha foi proposto para designar o ramo da química que se ocupa dos produtos químicos usados nas armas químicas e no terroris-
Dos três termos coloridos, só QV ("Green Chemistry") é sancionado pela Encyclopedia Brittanica [19].

Biotecnologia Verde, Vermelha, Preta, Azul e Branca. As cores verde, vermelha e preta são usadas em biotecnologia com significados diferentes dos usados em química; além disso, a biotecnologia usa também as cores azul e branca. As designações coloridas correntemente usadas neste ramo da ciência são $[20,21]:{ }^{5}$

Biotecnologia Verde - refere-se ao ramo da biotecnologia dirigido à agricultura e à alimentação;

Biotecnologia Vermelha - designa a actividade dirigida à medicina e saúde;

Biotecnologia Preta - é a biotecnologia das armas biológicas e bioquímicas, e do bioterrorismo;

Biotecnologia Azul - refere-se à actividade dirigida à aquacultura e exploração do mar;

Biotecnologia Branca - designa a biotecnologia industrial de fabrico de produtos químicos que, tal como a $\mathrm{QV}$, deve ser dirigida à protecção do ambiente e à promoção da Sustentabilidade.

Em suma, em biotecnologia o vermelho surge associado ao bem - à promoção da saúde humana - em contraste com o que sucede na química. Por outro lado, o verde tem em biotecnologia uma conotação directa com as plantas agrícolas enquanto que em QV a conotação à fitosfera é mais difusa e apenas histórica, como se referiu atrás - presentemente, está mais ligado à promoção do Desenvolvimento Sustentável.

Estas discrepâncias no uso das cores prestam-se naturalmente a confusões; por exemplo, quando se considera o Sétimo Princípio da QV ("Uso de matérias primas renováveis") [22] e o conceito de Biorrefinaria que dele resulta (por exemplo, ref. [23]), entram em jogo actividades com um mesmo objectivo mas de cores diferentes - a Biotecnologia Branca e a QV.

Da Revolução Verde à Química Verde. O termo Revolução Verde ${ }^{6}$ é vulgarmente usado no campo da história da agricultura para designar a mudança da prática agrícola realizada a partir da Segunda Grande Guerra Mundial que, por aliança da química (fertilizantes, pesticidas, etc.) com a biologia agrícola (apuramento das espécies, por exemplo, milhos híbridos), permitiu aumentar muito a produtividade agrícola e eliminar a fome na Terra (a menos de problemas de distribuição de alimentos) - tendo a cor verde, outra vez, uma conotação directa com a fitosfera. No entanto, as práticas de agricultura "industrializada" que possibilitaram a Revolução Verde, e que envolvem o uso intensivo de produtos químicos (adubos sintéticos, produtos fitossanitários, etc.), conduziram a numerosos problemas de poluição - facto que contribuiu para a emergência, nos anos setenta, do ambientalismo. Afinal, do ponto de vista químico, a Revolução (agrícola) Verde não foi tão verde quanto o nome sugere, quando é considerado o seu efeito ambiental: criou problemas diversos de poluição e de dispersão de vestígios de substâncias sintéticas no ambiente - e acabou por contribuir fortemente para que a sociedade to- 
masse consciência dos problemas de poluição e, mais tarde, para que os químicos sentissem a necessidade de mudar as suas práticas para a QV.

Utilização cultural da cor verde. Recentemente, num artigo sobre a história da QV [24], foi notado que a cor verde é usada pelas diversas civilizações como símbolo de sentimentos e posturas muito diversas e, frequentemente, em situações discrepantes [24,25]. Embora não seja adequado entrar aqui em detalhes, é pertinente notar que, afinal, as contradições no uso social do verde são frequentes - e naturalmente a discrepância no uso da cor pela química e biotecnologia terá de ser aceite (é apenas mais uma!).

\section{Conclusão}

A utilização das cores verde e vermeIho pela química é a da tecnologia das luzes de tráfico - verde para avançar, vermelho para proibir o avanço - ao contrário do que sucede com a biotecnologia, que usa um código de cores mais ligado à natureza (nomeadamente à biologia): o verde das plantas para a agricultura e alimentação e o vermelho do sangue humano para a medicina e saúde.

Para a opção de cores "tecnológica" da química poderão ter contribuído dois factos. Primeiro, a química estar bem inserida na civilização tecnológica, já que desempenhou um papel importante ao longo do desenvolvimento da Revolução Industrial. ${ }^{7}$ Segundo, a mentalidade técnica, pragmática, dos químicos industriais e engenheiros químicos adquirida neste contexto. Estes factos resultam provavelmente de a química ser a única ciência que estabeleceu uma indústria própria, a Indústria Química, e que tem associado um ramo de engenharia tradicional privativo, a Engenharia Química - o que foi uma consequência de, no século XIX, a química e a respectiva indústria se terem desenvolvido em paralelo e de uma forma integrada [26].

Em contraste, a biotecnologia, apesar de ser tão antiga como a química [27, 28], manteve até meados do sécu- lo XX uma actividade industrial de prática quase artesanal - por exemplo, de fabrico continuado no tempo apenas de um número limitado de produtos (etanol e ácido acético, fermentos de panificação, e depois, no início do século $X X$, ácidos cítrico e láctico) [29], e com escalamentos menores que na química. À medida que se desenvolveu, a biotecnologia recorreu crescentemente à tecnologia química (excepto quanto a reactores, muito específicos) e continua a ser implementada, em muitos casos, no âmbito da Indústria Química. Deste percurso resultou para a biotecnologia uma mentalidade "mais bio" e "menos tecno", de que ainda não se libertou completamente (e que talvez até se tenha acentuado com o uso crescente da engenharia genética). Em suma, o envolvimento mais limitado da tecnologia no desenvolvimento da biotecnologia, bem como este ter sido muito mais embutido no da civilização humana [28], ${ }^{8}$ terá determinado uma distinção cultural que pode explicar a diferença de atitude quanto à adopção das cores - esta resulta das mentalidades "mais biológica" da biotecnologia e "mais tecnológica" da química. ${ }^{9}$

Duas observações finais. Primeira, a ser verdadeira esta hipotética explicação para a génese tecnológica do código de cores "luzes de tráfico" (verde/ /vermelho) usado pela química, seria mais um exemplo de como a tecnologia pode condicionar a história [30] - embora este seja um exemplo menor, já que envolve apenas uma questão de linguagem. Segunda, como os códigos de cores da química e da biotecnologia têm fundamento cultural, será muito difícil promover mudanças que conduzam a uma unificação - pelo que vale a pena prevenir os leitores das diferenças, esperando evitar-lhes eventuais equívocos.

\section{Notas}

${ }^{1}$ A preferência pelo termo $Q V$ relativamente a outros que envolvem qualificativos referentes à Sustentabilidade, mas são menos incisivos, foi abordada em [31].

${ }^{2} \mathrm{O}$ verde continua a ser usado para adjectivar atitudes, movimentos, associações, partidos, etc., com objectivos programáticos que são dirigidos à melhoria da quali- dade do ambiente - mas as posturas destas actividades ou instituições quanto a processos para cumprir os seus objectivos têm evoluído significativamente, ou seja, o tom de verde tem mudado.

${ }^{3}$ Num artigo recente (2004) [32] em que se analisa a penetração da QV na indústria dos EUA com base em patentes registadas naquele país até 2001 sobre o tema, mostra-se que o número de patentes concedidas por ano cresceu rapidamente no período 1991-1993, semelhantemente à situação mostrada pela Fig.1 - mas quase uma década antes da revelada pela literatura científica e pedagógica. O crescimento poderá ter surgido em resposta à publicação da legislação ambiental nos fins dos anos oitenta, que aumentou os requisitos de protecção do ambiente a cumprir pelas empresas. No referido estudo, as patentes sobre QV foram identificadas pela inclusão no título, resumo, etc., de termos representativos de tecnologia química verde (por exemplo, "design de químicos mais seguros", "processos sem solventes", "matérias-primas alternativas", etc.) - não tendo sido encontrada, porém, qualquer patente com o termo QV no título, resumo ou lista de direitos patenteados. Em face disto, a comparação com os resultados na Fig. 1 pode não ser lícita.

${ }^{4}$ A natureza dual é característica de todas as ciências experimentais e das tecnologias - podem ser usadas para implementar o bem e o mal, dependendo de quem as pratica.

${ }^{5}$ Esta classificação parece ser a mais largamente aceite, embora haja uma proposta recente (2004) que envolve uma classificação mais fina dos campos e dez cores (o arco-íris não chega!), nem todas coincidentes com as apresentadas aqui [33].

6 O mentor da Revolução Verde foi Norman Borlaug (1914-...), Nobel da Paz (1970), que foi biografado recentemente [34] de uma forma interessante e elucidativa quanto àquela revolução e respectivo alcance.

${ }^{7}$ A profunda inserção é comprovada por a sociedade, ainda agora, mesmo quando os produtos químicos são "sujos" e nocivos para o ambiente, pressionar a continuação do seu uso...até que os químicos lhes proporcionem substitutos mais benignos.

${ }^{8}$ Recorde-se, por exemplo, a importância para os humanos que o vinho, cerveja e outras bebidas alcoólicas, obtidas por fermentação, sempre tiveram ao longo da civilização.

9 Esta mentalidade tecnológica foi sendo gradualmente perdida pelos químicos académicos ao longo do século $X X$, à medida que o avanço da química como ciência foi 
permitindo sintetizar cada vez mais moléculas não naturais - este facto corresponde a uma separação de escalas de praticar a química, da macroscópica para a molecular, o que contribuiu para criar um fosso entre a Química Académica (ou Laboratorial) e a Química Industrial, que ficaram separadas pela chamada "fronteira molecular" [35]. Uma pequena fracção destas moléculas sintéticas chegou à produção industrial, após elaborados estudos de escalamento para passar a fronteira, mas, mais tarde, muitos deste compostos revelaram-se incompatíveis com o ambiente e a biosfera - surgiram variados problemas de poluição por resíduos de compostos sintéticos e de outros usados no seu fabrico, que não foram previstos atempadamente aquando da síntese e da montagem do fabrico industrial. O desenvolvimento da $Q V$, cujo objectivo último é eliminar de raiz estes problemas, passa pela eliminação do referido fosso - por aproximação da Química Académica (químicos laboratoriais) e da Química Industrial (engenheiros químicos e químicos de processo), conforme defendido, em 2003, no chamado relatório Breslow \& Tirrell [35].

\section{RefERÊNCIAS}

[1] P. T. Anastas e C. T. Farris (eds.), "Benign by Design: Alternative Synthetic Design for Pollution Prevention", ACS Symp. Series 577 (1994).

[2] P. T. Anastas e T. C. Williamson (eds.), "Green Chemistry: Designing Chemistry for the Environment", ACS Symp. Series 626 (1996).

[3] S. C. DeVito e R. L. Garrett (eds.), "Designing Safer Chemicals: Green Chemistry for Pollution Prevention", ACS Symp. Series 640 (1996).

[4] P. T. Anastas, L. G. Heine e T. C. Williamson (eds.), "Green Engineering", ACS Symp. Series 766 (2000).

[5] P. T. Anastas, L. G. Heine e T. C. Williamson (eds.), "Green Chemical Synthesis and Processes", ACS Symp. Series 767 (2000).

[6] R. L. Lankey e P. T. Anastas (eds.), "Advancing Sustainability through Green Chemistry and Engineering", ACS Symp. Series 823 (2002).

[7] W. M. Nelson (ed.), "Agricultural Applications in Green Chemistry", ACS Symp. Series 886 (2004).

[8] P. T. Anastas e M. M. Kirchhoff, "Origins, Current Status, and Future Challenges of Green Chemsitry", Acc. Chem. Res. 35 (2002) 686-694.
[9] E. J. Woodhouse e S. Breyman, "Green Chemistry as Social Movement?", Sci. Techn.\& Hum. Values 30 (2005) 199-222 .

[10] R. Sheldon, "Green Chemistry - One Year On", Green Chem. 2 (2000) G1-G4.

[11] I. Amato, "The Slow Birth of Green Chemistry", Science 259 (1993) 15381541.

[12] T. C. Collins, "Introduction Green Chemistry in Teaching and Research", J. Chem. Ed. 72 (1995) 965-969.

[13] Green Chemistry, http://www.rsc.org/ Publishing/Journals/gc/index.asp

[14] ACS, Advanced Article Search, em http://pubs.acs.org/wls/journals/query/subscriberSearch.html (acedida em 09/12/2007).

[15] JCEd, Index Advanced Search, em http://jchemed.chem.wisc.edu/Journal/Search/search.html (acedida em 09/12/2007).

[16] RSC Publications, Journals, em http://www.rsc.org/Publishing/Journals/fjournalsearch.asp (acedida em 09/12/2007).

[17] ScienceDirect, em http://www.sciencedirect.com/ (acedida em 10/12/2007)

[18] S. E. Manahan, Environmental Chemistry, $8^{a}$ ed., CRC Press (2005) p. 744.

[19] Encyclopedia Brittanica - Deluxe Edition, CD (2005)

[20] EuropaBio, White Biotecnology: Gateway to a More Sustainable Future (2003) p. 7

[21] R. Wedin, "Chemistry on a High-carb Diet", Chemistry (ACS), Spring (2004) 23-27.

[22] P. T. Anatas e J. C. Warner, Green Chemistry - Theory and Practice, Oxford UP (1998).

[23] B. Kamm, P. R. Gruber e M. Kamm (eds.), Biorefineries - Industrial Processes and Products, 2 Vol., Wiley$\mathrm{VCH}(2006)$

[24] P. Tundo e F. Aricò, "Green Chemistry on the Rise - Thoughts on the Short History of the Field", Chemistry Int. 29(5) (2007) 4-7.

[25] Green, em http://encyclopedia.thefreedictionary.com/green (acedido em 8/12/2007)

[26] J. V. Koleske, "Economic Aspects of the Chemical Industry", em J. A. Kent (ed), Riegel's Handbook of Industrial Chemistry, $10^{\mathrm{a}}$ ed., Kluwer (2003) p. 1.

[27] P. E. McGovern et al, "Fermented Beverages of Pre- and Proto-Historic China", Proc. Nat. Ac. Sci. (US) 101 (2004) 17593-17598.

[28] E. J. DaSilva, "Art, Biotechnology and the Culture of Peace", Electronic J. Biotechn. (on line) 7(2)(2004), em http://www.ejbiotechnology.info/content/vol7/issue2/full/8/8.pdf (acedido em 8/12/2007).

[29] P. F. Stanbury, A. Whitaker e S. J. Hall, Principles of Fermentation Technology, $2^{\mathrm{a}}$ ed, Butterworth (2003) p. 6.

[30] M. R. Smith e L. Marx (eds.), Does Technology Drive History? The Dilemma of Technological Determinism, MIT Press (1994).

[31] A. A. S. C. Machado, "Química e Desenvolvimento Sustentável - QV, QUIVES, QUISUS", Química-Bol. SPQ 95 (2004), 59-67.

[32] T. J. Namerroff, R. J. Garant e M. B. Albert, "Adoption of Green Chemistry: An Analysis Based on US Patents", Research Policy 33 (2004) 959-974.

[33] E. J. DaSilva, "The Colours of Biotechnology: Science; Development and Humankind", Electronic J. of Biotechn. (on line) 7(3) (2004), em http://www. ejbiotechnology.info/content/vol7/ issue3/editorial.html (acedido em 8/12/2007).

[34] L. Hesser, The Man Who Fed the World - Nobel Peace Prize Laureate Norman Borlaug and His Battle to End World Hunger, Durban House (2006).

[35] R. Breslow e M. V. Tirrell (eds.), Committee on Challenges for the Chemical Sciences in the 21st Century (US National Research Council), Beyond the Molecular Frontier - Challenges for Chemistry and Chemical Engineering, National Academy Press (2003). 


\section{Where Chemistry Meets Life Science}

\section{4 national chemical \\ societies of EUChemSoc \\ *} are co-owners of ChemBioChem

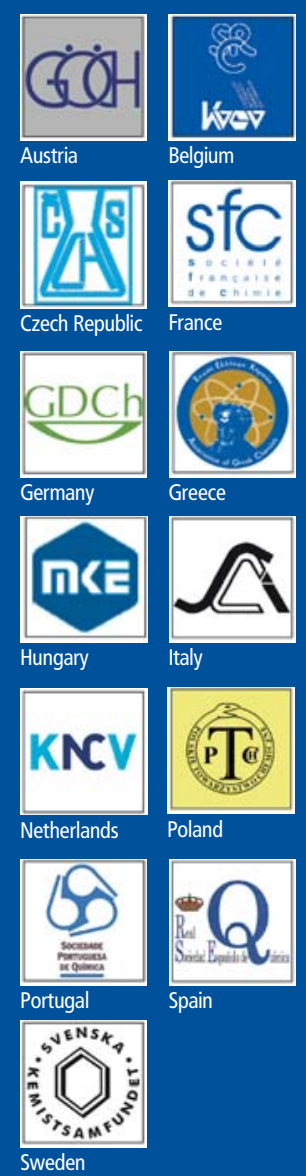

Subscribe now!

For further information and to subscribe please send an E-mail to:

subinfo@wiley.com (USA/Canada)

service@wiley-vch.de (Germany/Austria/Switzerland) cs-journals@wiley.co.uk (all other areas)

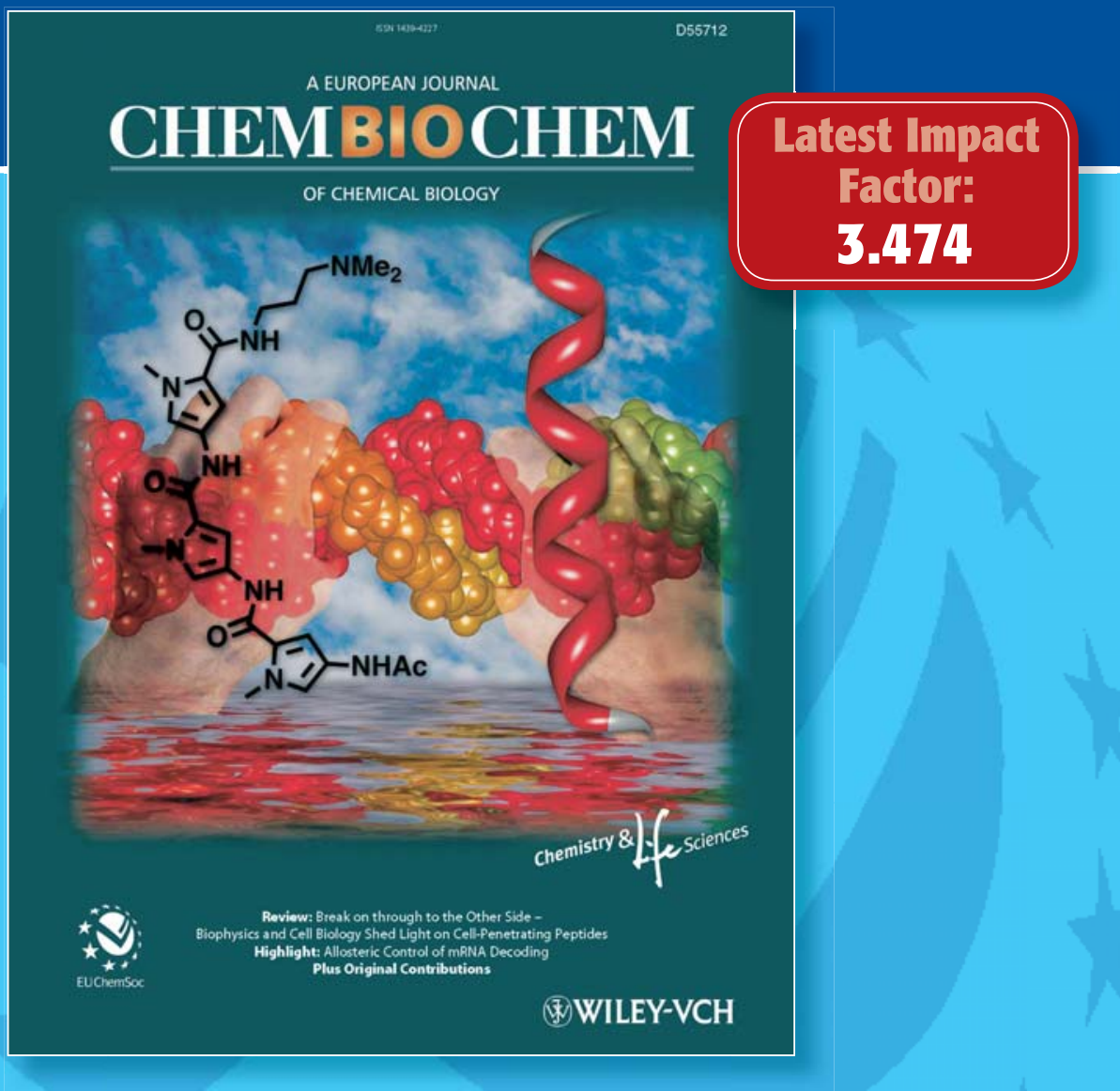

an attractive mixture of:

Chairmen of the Editorial Board: Alan R. Fersht (UK)

- Short communications Jean-Marie Lehn (France)

- Full papers

- Reviews \& Minireviews

Editor:

Peter Gölitz

- Highlights \& Concepts Managing Editor:

- News \& Comments

- Book and Multimedia Lisa Abel

Reviews

\section{Visit ChemBFoChem online} wuw.chembiochem-or:

$\underbrace{\text { Interer }}_{\text {DISCOVER SOMETHING GRAT }}$ WILEY-VCH 


\section{Actualidades Científicas}

\section{BiocombustíveIS E EFEITO DE ESTUFA}

O número de 29 de Fevereiro da revista americana Science divulga dois artigos relativos ao uso de biocombustível para a redução das emissões de dióxido de carbono, que colocam algumas reservas na prossecução deste objectivo.

A procura de alternativas ao petróleo tem conduzido a um aumento da produção de biocombustível a partir de produtos alimentares, como milho, soja e cana de açúcar.

A biomassa dos solos e das plantas constitui o maior reservatório biologicamente activo de carbono terrestre, contendo cerca de 2,7 vezes mais carbono do que a atmosfera. Como o cultivo de matéria-prima para a produção de biocombustível remove dióxido de carbono da atmosfera, o biocombustível pode, em teoria, reduzir a quantidade de gases causadores de feito de estufa (como dióxido de carbono e metano) face à utilização de combustíveis fósseis. Contudo, estes estudos não tiveram em consideração as emissões de carbono que ocorrem em consequência de, para responderem à subida de preços, os agricul- tores converterem floresta e terrenos de pasto em novos terrenos agrícolas para compensar o desvio de produtos para a produção de biocombustível.

Num primeiro estudo, dirigido por David Tilman da Universidade do Minesotta (EUA), foi avaliado o efeito do tipo de terrenos agrícolas usados no cultivo de produtos para biocombustível sobre a quantidade de dióxido de carbono produzida. Em particular, foi concluído que a utilização de terrenos resultantes da conversão de florestas tropicais, terrenos de turfa, savanas ou terrenos de pasto para cultivar produtos para o fabrico de biocombustível no Brasil, Sudeste da Ásia e Estados Unidos resulta na libertação de 17 a 420 vezes mais dióxido de carbono do que as reduções anuais dos gases de efeito de estufa que serão conseguidas pela substituição dos combustíveis fósseis por este biocombustível. Apenas quando o biocombustível é produzido a partir de biomassa desperdiçada ou de biomassa crescida em terrenos agrícolas degradados ou abandonados este pode oferecer vantagens imediatas e sustentáveis em relação à produção dos gases causadores do efeito de estufa. Ou seja, a conversão de habitats ricos em carbono para a produção de biocombustível conduz a um aumento das emissões de dióxido de carbono por décadas ou séculos em relação às emissões causadas pela utilização de combustíveis fósseis.

Num segundo estudo (efectuado por uma equipa liderada por Timothy Searchinger da Universidade de Princeton) foi desenvolvido um modelo para estimar o efeito da utilização de etanol produzido a partir de cereais cultivados em terrenos convertidos. Os resultados mostram que, em vez de reduzir em $20 \%$, a utilização de etanol produzido nestes terrenos quase que duplica as emissões de gases causadores do efeito de estufa ao longo de cerca de 30 anos e aumenta os gases de efeito de estufa durante 167 anos.

Estes estudos realçam a necessidade de a produção de biocombustíveis se fazer acima de tudo a partir de desperdícios.

Finalmente acresce ainda que a crescente utilização de biocombustíveis está a afectar o preço dos produtos que, como o milho e a soja, são também usados na alimentação. $E$ este factor tem, necessariamente, que ser também tido em consideração. 